\title{
Tratamiento satisfactorio con fluconazol de la candidemia relacionada con el catéter por Candida haemulonii: informe sobre dos pacientes neonatales Successful fluconazole treatment of catheter-related candidemia caused by Candida haemulonii: report of 2 neonatal cases
}

Dra. Fang-Qin Xia ${ }^{a}$, Dr. Chu-Yuan Yea, Dra. Wei-Wei Xie ${ }^{a}$, Dra. Neha-Devi Poonit ${ }^{a}$ y Dr. Yong-Hai, Zhou

\section{RESUMEN}

La Candida haemulonii forma parte de la especie Candida no albicans. La candidemia por C. haemulonii es sumamente infrecuente, pero mortal, en los recién nacidos. Se informa sobre los dos primeros recién nacidos con candidemia por C. haemulonii en China tratados con fluconazol y se revisan dos artículos informados con anterioridad. Nuestro informe incrementa la sensibilización sobre la candidemia por C. haemulonii en recién nacidos críticos y resalta la importancia de un diagnóstico y un tratamiento tempranos de esta infección mortal.

Palabras clave: recién nacido; Candida haemulonii; candidemia.

http: / / dx.doi.org/10.5546/ aap.2018.e663

Texto completo en inglés:

http: / / dx.doi.org/10.5546/ aap.2018.eng.e663

Cómo citar: Xia FQ, Ye CY, Xie WW, et al. Tratamiento satisfactorio con fluconazol de la candidemia relacionada con el catéter por Candida haemulonii: informe sobre dos pacientes neonatales. Arch Argent Pediatr 2018;116(5):e663-e666. a. Departamento de Pediatría, Segundo Hospital Afiliado y Hospital de Niños Yuying del Colegio Médico Wenzhou, Wenzhou, China.

Correspondencia:Dr. Yong-HaiZhou:zhouyonghai311@126.com

Financiamiento: Ninguno.

Conflicto de intereses: Ninguno que declarar.

Recibido: 11-12-2017

Aceptado: 26-3-2018

\section{ANTECEDENTES}

La Candida haemulonii, de la especie Candida no albicans, se aisló por primera vez en un ser humano en $1984 .{ }^{1}$ La candidemia por C. haemulonii es sumamente rara, aunque mortal, en la unidad de cuidados intensivos neonatales (UCIN). ${ }^{2}$ Hasta el momento, Khan y col. y Silva y col. mencionaron los casos de cinco recién nacidos con candidemia por C. haemulonii (Tabla 1);, ${ }^{3,4} \mathrm{dos}$ de ellos fallecieron. Aquí informamos sobre los primeros dos recién nacidos con candidemia por C. haemulonii en China tratados con fluconazol y revisamos los cinco casos de los pacientes con esta infección notificados anteriormente en la bibliografía.

\section{PRESENTACIÓN DE LOS CASOS Caso 1}

Un varón de 12 días de vida fue derivado a nuestra UCIN desde un hospital municipal con antecedentes de distensión abdominal de dos días de evolución. Este bebé había nacido por parto vaginal a las $30^{+5}$ semanas de gestación debido a rotura prematura de membranas. Pesó $1520 \mathrm{~g}$ y tuvo un puntaje de Apgar de 5 en el minuto 1 y de 9 en el minuto 5. La madre era una mujer de 23 años, desempleada y con antecedentes de atención prenatal normal. Al bebé prematuro

TABLA 1. Casos de candidemia neonatal por C. haemulonii

\begin{tabular}{lcccc}
\hline Núm. & $\begin{array}{c}\text { Sexo/ } \\
\text { edad }\end{array}$ & $\begin{array}{c}\text { País/ } \\
\text { región }\end{array}$ & Desenlace & $\begin{array}{c}\text { Año de } \\
\text { publicación } \\
\text { [Referencia] }\end{array}$ \\
\hline 1 & $\mathrm{M} / 35 \mathrm{sem}$. & Kuwait & Fallecimiento & $2007^{3}$ \\
2 & $\mathrm{M} / 26 \mathrm{sem}$. & Kuwait & Sobrevida & $2007^{3}$ \\
3 & $\mathrm{~F} / 25 \mathrm{sem}$. & Kuwait & Sobrevida & $2007^{3}$ \\
4 & $\mathrm{~F} / 31 \mathrm{sem}$. & Kuwait & Fallecimiento & $2007^{3}$ \\
5 & $\mathrm{~F} / 26 \mathrm{sem}$. & Brasil & Sobrevida & $2015^{4}$ \\
6 Caso 1 & $\mathrm{M} / 30^{+5} \mathrm{sem}$. & China & Sobrevida & Nuestro caso \\
7 Caso 2 & $\mathrm{F} / 39^{+5} \mathrm{sem}$. & China & Sobrevida & Nuestro caso \\
\hline
\end{tabular}


se le realizó una intubación endotraqueal y se lo conectó a un respirador al momento de nacer debido a síndrome de dificultad respiratoria. Después de siete días, se interrumpió el uso del respirador y se inició la administración de presión positiva continua en las vías respiratorias por vía nasal. Al paciente se lo alimentaba por sonda nasogástrica y estaba creciendo adecuadamente. A los 10 días de vida, se observaron distensión abdominal y residuos de alimentos. En la radiografía, se detectó neumoperitoneo, y la proteína C-reactiva (PCR) había aumentado a $146 \mathrm{mg} / \mathrm{L}$. Por lo tanto, se ingresó al bebé a nuestra unidad. Poco tiempo después, el paciente padeció insuficiencia respiratoria y fue necesario conectarlo a un respirador. Luego, se confirmó la presencia de enterocolitis necrosante neonatal (ENN). Se lo operó de emergencia y se le extirparon $40 \mathrm{~cm}$ de intestino delgado necrótico. Posteriormente, se inició la administración de meropenem $(60 \mathrm{mg} / \mathrm{kg} /$ día, cada 8 horas) por vía intravenosa y alimentación parenteral por catéter central de inserción periférica (PICC, por sus siglas en inglés). Al quinto día de hospitalización en nuestra unidad, en el cultivo de líquido ascítico se observaron Pseudomonas aeruginosa y Aerobacter cloacae, pero el hemocultivo fue negativo y la temperatura era normal. Se desconectó al paciente del respirador al sexto día de la hospitalización y la PCR disminuyó a valores normales tras 29 días de hospitalización en la UCIN.

A los 36 días de hospitalización en nuestra unidad, la temperatura del bebé subió a $38,4^{\circ} \mathrm{C}$. En los análisis de laboratorio, se observaron una concentración de hemoglobina de $95 \mathrm{~g} / \mathrm{L}$, un recuento de leucocitos de $8,4 \times 10^{9} / \mathrm{L}(60,1 \%$ de neutrófilos) y un recuento de plaquetas de $76 \times 10^{9} / \mathrm{L}$. La PCR aumentó a $33 \mathrm{mg} / \mathrm{L}$. Se retiró la PICC. Se obtuvieron muestras de sangre y del extremo del catéter para realizar cultivos.

Después de 72 horas, en ambos cultivos se produjeron unidades formadoras de colonias indicativas de proliferación de levaduras, que luego se identificaron como C. haemulonii mediante tarjetas Vitek 2 Compact Yeast (bioMérieux, Francia). Se confirmó la identidad de la C. haemulonii por técnicas de biología molecular. ${ }^{3}$ En el fungigrama, se observó que la cepa de Candida aislada era sensible a la anfotericina B, al ketoconazol, al fluconazol, al itraconazol, al clotrimazol y a la nistatina. Se administró fluconazol en dosis de $6 \mathrm{mg} / \mathrm{kg} /$ día por vía intravenosa. Después de 30 días de tratamiento con fluconazol, la infección estaba controlada y el bebé se recuperó. A los 88 días de hospitalización, el paciente podía digerir $50 \mathrm{ml}$ de leche cada 3 horas y recibió el alta. En el seguimiento posterior, el bebé estaba creciendo normalmente.

\section{Caso 2}

Se presentó en nuestra UCIN una recién nacida con dificultad respiratoria y líquido amniótico meconial de grado III. Esta bebé había nacido a las $39^{+5}$ semanas de gestación por cesárea debido a sufrimiento fetal. Pesó $2650 \mathrm{~g}$ y tenía un puntaje de Apgar de 6 en el minuto 1 y de 8 en el minuto 5. La madre era una comerciante, de 27 años y con antecedentes de atención prenatal normal. Se conectó a la bebé al respirador y se inició la alimentación parenteral por catéter venoso umbilical.

A los 11 días de hospitalización, la temperatura de la bebé subió a $38,4{ }^{\circ} \mathrm{C}$. En los análisis de laboratorio, se observaron una concentración de hemoglobina de $169 \mathrm{~g} / \mathrm{L}$, un recuento de leucocitos de 15,7 × 10\% / L (57,9\% de neutrófilos) y un recuento de plaquetas de $39 \times 10^{9} / \mathrm{L}$. La PCR aumentó a $17 \mathrm{mg} / \mathrm{L}$. Por lo tanto, se retiró el catéter venoso umbilical. En los cultivos realizados en las muestras de sangre y del extremo del catéter se observaron algunas unidades formadoras de colonias indicativas de proliferación de levaduras, que luego se identificaron como C. haemulonii mediante tarjetas Vitek 2 Compact Yeast (bioMérieux, Francia). Se confirmó la identidad de la C. haemulonii por técnicas de biología molecular. ${ }^{3}$ En el fungigrama, se observó que la cepa de Candida aislada era resistente al clotrimazol y a la nistatina, pero sensible a la anfotericina B, al ketoconazol, al fluconazol $\mathrm{y}$ al itraconazol. Se administró fluconazol en dosis de $6 \mathrm{mg} / \mathrm{kg} /$ día por vía intravenosa. Se desconectó a la paciente del respirador a los 16 días de hospitalización. Después de 26 días de tratamiento con fluconazol, la bebé se recuperó. A los 38 días de hospitalización, recibió el alta después de que se obtuvieron resultados negativos en dos hemocultivos consecutivos. Durante el seguimiento posterior, la bebé estaba creciendo normalmente.

\section{DISCUSIÓN}

En los últimos años, se ha observado que las especies de Candida representan patógenos intrahospitalarios emergentes importantes. Se ha informado que la Candida haemulonii, un tipo de Candida, causa fungemia, ${ }^{3-11}$ osteítis, ${ }^{12}$. ${ }^{2}$ eritonitis ${ }^{13}$ 
y otitis media crónica. ${ }^{8}$ Hasta el momento, se describió candidemia por C. haemulonii en cinco recién nacidos, de los cuales, dos $(40,0 \%)$ fallecieron. En la Tabla 1, se resumen las características relevantes de los cinco pacientes con candidemia por C. haemulonii informados. Los casos de dos recién nacidos con candidemia por C. haemulonii que describimos aquí son los primeros en China.

La fiebre es el signo inicial más frecuente. Es difícil diagnosticar la infección por $C$. haemulonii en las primeras etapas debido a los signos y síntomas inespecíficos. Cabe señalar que se detectó trombocitopenia en nuestros dos pacientes, lo que podría ser una indicación de candidemia por C. haemulonii.

Según los informes previos, los factores de riesgo probables de la candidemia por C. haemulonii incluyen el uso extendido de antibióticos de amplio espectro, ${ }^{6}$ el uso de dispositivos terapéuticos (por ej., catéteres y respiradores) ${ }^{6-10}$ haber sido prematuro, ${ }^{3,4}$ ser paciente oncológico, ${ }^{4,7,9,11}$ las técnicas quirúrgicas novedosas ${ }^{5}$ y la insuficiencia renal crónica. ${ }^{9}$

En este informe, el caso 1 corresponde a un bebé prematuro sometido a una cirugía por ENN que recibió antibióticos de amplio espectro. El paciente estuvo conectado al respirador y se le colocó un PICC. El período de permanencia en la UCIN fue prolongado. Estos fueron los factores de riesgo de candidemia por $C$. haemulonii en este paciente. A pesar de haber nacido a término, la paciente del caso 2 también tenía factores de riesgo de candidemia por $C$. haemulonii; se la conectó al respirador y se le colocó un catéter venoso umbilical. El uso a largo plazo de un catéter para hiperalimentación fue el factor de riesgo asociado para candidemia, y el cultivo positivo de los extremos del catéter era indicativo de una candidemia relacionada con el catéter.

La anfotericina B, el ketoconazol, el fluconazol, el itraconazol y el clotrimazol son los antibióticos comúnmente disponibles para el tratamiento de las micosis graves. No obstante, se ha informado que la $C$. haemulonii es resistente a la anfotericina $\mathrm{B}, 3,4,6,11$ al fluconazol, ${ }^{3,7-10}$ al itraconazol, ${ }^{3,7,8}$, a la flucitosina ${ }^{11} \mathrm{y}$ a la micafungina. ${ }^{5} \mathrm{Zia}$ y col., describieron los casos de cuatro pacientes en quienes las cepas de Candida aisladas eran resistentes a la anfotericina $B$, al fluconazol y al itraconazol; dos de ellos fallecieron. ${ }^{3}$ En nuestro estudio, se trató a los pacientes con fluconazol y se recuperaron. Las cepas de Candida aisladas en nuestros pacientes eran sensibles a la anfotericina B y al fluconazol. Con base en estos resultados, se sospechó que la multirresistencia, en especial, la resistencia a la anfotericina $B$, al fluconazol y al itraconazol, podría ser una de las causas más relevantes de muerte en los pacientes con candidemia por $C$. haemulonii. La resistencia de C. haemulonii representa un desafío en el tratamiento de la candidiasis.

A modo de conclusión, la candidemia por C. haemulonii es sumamente infrecuente, pero mortal, en la UCIN. Las manifestaciones clínicas de la candidemia por $C$. haemulonii en las primeras etapas son similares a las de las infecciones comunes. Según nuestros hallazgos, cuando se detectan fiebre y trombocitopenia en un recién nacido con una enfermedad subyacente o dispositivos terapéuticos invasivos (por ej., PICC o catéter venoso umbilical), se debe sospechar la presencia de candidemia por $C$. haemulonii. La resistencia de $C$. haemulonii representa un desafío en el tratamiento. Es fundamental la administración de antimicóticos a los que la cepa sea sensible y la remoción del PICC o del catéter venoso umbilical desde un principio.

\section{REFERENCIAS}

1. Lavarde V, Daniel F, Saez H, et al. Peritonite mycosique a Torulopsis haemulonii. Bul Soc Fr Mycol Med 1984; 13:173-6.

2. Panackal AA. Optimizing containment and control of Candida parapsilosis fungemia among neonates in the outbreak setting using a mathematical modeling approach. J Mycol 2013; 2013:413874.

3. Khan ZU, Al-Sweih NA, Ahmad S, et al. Outbreak of fungemia among neonates caused by Candida haemulonii resistant to amphotericin $\mathrm{B}$, itraconazole, and fluconazole. $J$ Clin Microbiol 2007; 45(6):2025-7.

4. Silva CM, Carvalho-Parahym AM, Macêdo DP, et al. Neonatal Candidemia Caused by Candida haemulonii Case Report and Review of Literature. Mycopathologia 2015;180(12):69-73.

5. Muro MD, Motta Fde A, Burger M, et al. Echinocandin resistance in two Candida haemulonii isolates from pediatric patients. J Clin Microbiol 2012; 50(11):3783-5.

6. Rodero L, Cuenca-Estrella M, Córdoba S, et al. Transient fungemia caused by an amphotericin B-resistant isolate of Candida haemulonii. J Clin Microbiol 2002; 40(6):2266-9.

7. Giusiano G, Mangiaterra M, Garcia Saito V, et al. Fluconazole and itraconazole resistance of yeasts isolated from the bloodstream and catheters of hospitalized pediatric patients. Chemotherapy 2006; 52(5):254-9.

8. Kim MN, Shin JH, Sung $\mathrm{H}$, et al. Candida haemulonii and closely related species at 5 university hospitals in Korea identification, antifungal susceptibility, and clinical features. Clin Infect Dis 2009; 48(6):e57-61.

9. RuanSY,Kuo YW, HuangCT, et al. Infections due to Candida haemulonii species identification, antifungal susceptibility and outcomes. Int J Antimicrob Agents 2010; 35(1):85-8.

10. Kim S, Ko KS, Moon SY, et al. Catheter-related candidemia caused by Candida haemulonii in a patient in long-term hospital care. J Korean Med Sci 2011; 26(2):297-300. 
e666 / Arch Argent Pediatr 2018;116(5):e663-e666 / Presentación de casos clínicos

11. Almeida JN Jr, Motta AL, Rossi F, et al. First report of a clinical isolate of Candida haemulonii in Brazil. Clinics (Sao Paulo) 2012; 67(10):1229-31.

12. CrouzetJ, Sotto A, Picard E, etal. A case of Candida haemulonii osteitis clinical features, biochemical characteristics, and antifungal resistance profile. Clin Microbiol Infect 2011; 17(7):1068-70.

13. Yuvaraj A, Rohit A, Koshy PJ, et al. Rare occurrence of fatal Candida haemulonii peritonitis in a diabetic CAPD patient. Ren Fail 2014; 36(9):1466-7. 